\title{
Distributed Consensus Protocols for Coordinating Buyers
}

\author{
Dario Bauso*, Laura Giarré*, Raffaele Pesenti ** \\ * DIAS, Universitá di Palermo, Italy, e-mail: bauso@ias.unipa.it, giarre@unipa.it \\ ** DINFO, Universitá di Palermo, Italy, e-mail: pesenti@unipa.it
}

\begin{abstract}
In this paper, we introduce a distributed consensus protocol for coordinating orders of a network of buyers also called agents/decision makers. Each buyer chooses a different threshold strategy, defining its intention to place an order only if at least other $l$ buyers will do the same. We prove that consensus is reached asymptotically globally and coordination is the same that if the decision making process would be centralized, namely, any decision maker (DM) has access to the thresholds of all other DMs and chooses to order or not. The proposed distributed protocol has the advantage that buyers do not have to communicate their threshold strategy in advance, and consensus is reached without exploring all the possible threshold values.
\end{abstract}

\section{INTRODUCTION}

Coordination of a team of agents/vehicles is an important task in several applications including inventory routing [3], formation flight of unmanned air vehicles (UAV's) [6], attitude alignment of underwater vehicles, and congestion/flow control in communication networks [4].

All these types of problems can be addressed in the context of cooperative and distributed control systems. For such problems, the lack of a centralized information processing places emphasis on the role of a number of distributed protocols for the information propagation.

In [1] a decentralized bang-bang continuous time control law is proposed that track a desired state for a failure prone network in presence of unknown but bounded disturbances.

In a particular type of cooperative and distributed problems, known as agreement or consensus problems [7], it is requested the eventual convergence of all the node states to a common decision-value a-priori unknown.

A central point in consensus problems is the connection between the graph structure, and delays or distortions in communication links [2].

In this paper we consider a group of buyers (in the following also called either agents or decision makers-DMs) aiming at coordinating their daily ordering decisions. Such a coordination is generally motivated by the possibility of sharing fixed transportation costs. We will express coordination requirements for each buyer in terms of a minimum threshold on the number of buyers, $l$, to coordinate orders with. Here, each threshold $l_{i}$ is assumed constant and a-priori known only to the corresponding $i^{t h}$ agent. In this context, we are interested in defining a protocol for information interchange among buyers such that the number of active buyers, i.e. the buyers that eventually place orders, on the bases of the available information is maximized.
We will show that it can be trivially defined a centralized protocol such that the number of active buyers can be maximized. More interestingly, we will prove that the same maximal set of active buyers can be achieved by means of a distributed protocol. In particular, we will show that it is sufficient that each agent communicates to a subset of other agents its current local estimation of the possible percentage of active buyers.

\section{Problem Statement}

In this section some notation is initially introduced and hence the problem dealt with in this paper is formally stated.

- Consider a network $\mathcal{G}=(\mathcal{V}, \mathcal{E})$; each buyer is a node $v_{i} \in \mathcal{V}$, where $i=1,2, \ldots, n$, and each communication link is an edge $e=\left(v_{i}, v_{j}\right) \in \mathcal{E} ; i, j=1,2, \ldots, n$. Let $n=|\mathcal{V}|$, where $|S|$ indicates the cardinality of the set $S$.

- Let the state of the $i^{t h}$-node be $x_{i} \in \Re^{m}$, i.e., the local information vector; denote with $x=\left\{x_{i}\right\}$ the network state, i.e., the collection of the states of all the nodes.

- Let the information vector transmitted by the $i^{\text {th }}$-node be $y_{i} \in \Re^{q}$; let the vector $y=\left\{y_{i}\right\}$ be the collection of the information transmitted by all nodes.

- Consider a protocol $\Pi=\left\{\left(f_{i}, h_{i}, \phi_{i}\right): i=1,2, \ldots, n\right\}$, where, for each $i$,

- $f_{i}: \Re^{q n} \times \Re^{m} \rightarrow \Re^{m}$ describes the dynamics of the state of the $i^{\text {th }}$-node as a function of the information both available at the node itself and transmitted by the other nodes $\left(\dot{x}_{i}=f_{i}\left(y, x_{i}\right)\right)$

- $h_{i}: \Re^{m} \longrightarrow \Re^{q}$ generates the transmitted information vector $y_{i}$ given the state node $x_{i}$ (i.e., $\left.y_{i}=h_{i}\left(x_{i}\right)\right)$

- $\phi_{i}: \Re^{m} \longrightarrow \Re$ estimates the number of active buyers given the information vector $x_{i}$.

- Associate to protocol $\Pi$ the set of the eventually active buyers $A_{\Pi}=\left\{i \in \mathcal{V}: \phi_{i}\left(\bar{x}_{i}\right) \geq l_{i}\right\}$, where, for each $i, \bar{x}_{i}$ is the final value, if it exists, of the state of the $i^{t h}$-node given the protocol $\Pi$ and $l_{i}$ is the threshold value of the $i^{\text {th }}$-buyer as defined in the introduction. We conventionally set $l_{i}=\infty$ if the $i^{t h}$-buyer is not interested in ordering whichever the number of active buyer is.

\section{Definition:}

In this context, we define as decentralized/distributed protocol, a protocol in which, for each $i \in \mathcal{V}, f_{i}$ is a function only 
of the transmitted information vectors of the nodes in the neighborhood $J_{i}$ of $i$, where $J_{i}=\{j:(i, j) \in \mathcal{E}\} \cup\{i\}$, i.e., the set all the agents with which $i$ is connected and $i$ itself. Let us make the two following assumptions:

Assumption 1: information about the total number of agents in the network, $n$, is shared by all agents

Assumption 2: At the beginning, each agent assumes its threshold strategy is chosen by all other agents.

Let us now formally state the problem under concern. Problem 1

Given a set of buyers reviewed as dynamic agents of a network with topology $\mathcal{G}=(\mathcal{V}, \mathcal{E})$. Let characterize the generic $i^{\text {th }}$ node by the threshold $l_{i}$. Given Assumptions $1-2$, determine a distributed protocol $\Pi$ which maximizes the set of the active buyers $A_{\Pi}$ and minimizes the dimensions of both the node states $m$ and the transmitted information vector $q$.

In the rest of the paper it is proved that a distributed protocol exists which is an optimal solution for the above problem, i.e., it optimizes all the three stated objectives at the same time. In the following subsection an upper bound for $A_{\Pi}$ is trivially determined. Then, in Section IV, we define an optimal distributed protocol.

\section{Preliminary Results}

In this section an upper bound for $A_{\Pi}$ is determined. To this aim, let us define $P_{l}=\left\{i \in \mathcal{V}: l_{i} \leq l\right\}$ the subset of agents that would place an order if at least $l-1$ other buyers would do the same.

Definition:

Define $S \in \mathcal{V}$ as a compatible set iff $\forall i \in S$ it is such that $l_{i} \leq|S|$. In other words, a compatible set $S$ of buyers is a set of buyers that would order if they were sure that all the other agents in $S$ would do the same.

Let $A=\max \{S: S$ is compatible $\}$ the maximal set of possible active buyers.

\section{Theorem 1}

It holds $A=P_{\tilde{l}}$ where $\tilde{l}$ is defined by the following condition

$$
\tilde{l}=\max \left\{l \in 1, \ldots, n:\left|P_{l}\right|=l\right\} .
$$

Proof: We prove first that $A=P_{|A|}$

1) $A \supseteq P_{|A|}$ Maximality and compatibility of $A$ leads to $A \supseteq\left\{i: l_{i} \leq|A|\right\}=P_{|A|}$. The second equality comes from the definition itself of $P_{|A|}$.

2) $A \subseteq P_{|A|}$ Let $\{\bar{l}\}=\max \left\{l_{i}: i \in A\right\}$. Then it holds, $\bar{l} \leq|A|$. Hence, $A \subseteq\left\{l_{i} \leq \bar{l}\right\} \subseteq\left\{i: l_{i} \leq|A|\right\}=P_{|A|}$

Let us observe that $\left|P_{|A|}\right|=|A|$. Because of (1) and observing $P_{l} \subseteq P_{l+1}$ for the definition itself of $P_{l}$, it holds $|A| \leq \tilde{l}$ that yields $P_{|A|} \subseteq P_{\tilde{l}}$. Since $P_{\tilde{l}}=\left\{i \in \mathcal{V}: l_{i} \leq \tilde{l}\right\}=\{i \in$ $\left.\mathcal{V}: l_{i} \leq\left|P_{\tilde{l}}\right|\right\}$ then $P_{\tilde{l}}$ is compatible and therefore for the maximality of $A,|A| \geq\left|P_{\tilde{l}}\right|=\tilde{l}$. Then, $\tilde{l} \leq|A| \leq \tilde{l}$ from which $A=P_{\tilde{l}}$.

Given Theorem 1 it can be verified that exists at least a centralized protocol $\hat{\Pi}$ that yields $A_{\hat{\Pi}}=A$.
On the purpose, define $\hat{\Pi}$ as follows: for each $i \in \mathcal{V}$,

$$
\left\{\begin{aligned}
h_{i}\left(x_{i}\right)= & \text { const }=l_{i} \\
f_{i}\left(y, x_{i}\right)= & \delta(t) \max \{l \in\{1, \ldots, n\}: \\
& \left.\sum_{i \in \mathcal{V}} 1\left(y_{i} \leq l\right)=l\right\} \\
\phi\left(x_{i}\right)= & x_{i} .
\end{aligned}\right.
$$

To finally show that $A_{\hat{\Pi}}=A$ observe that given the above defined protocol each node $i$ transmits the information $y_{i}=$ $l_{i}$, and assumes the state $x_{i}=\max \{l \in\{1, \ldots, n\}$ : $\left.\sum_{i \in \mathcal{V}} 1\left(y_{i} \leq l\right)=l\right\}$, where $\sum_{i \in \mathcal{V}} 1\left(y_{i} \leq l\right)$ simply counts the nodes whose threshold is less than or equal to $l$, and hence, $\sum_{i \in \mathcal{V}} 1\left(y_{i} \leq l\right)=\left|P_{l}\right|$ which in turns implies, due to (1), $x_{i}=\tilde{l}=\phi_{i}\left(x_{i}\right)$. Then $A_{\hat{\Pi}}=\left\{i \in \mathcal{V}: \tilde{l} \geq l_{i}\right\}$ which, by definition, is equal to $P_{\tilde{l}}=A$.

In the following section we will show that it exists also a decentralized protocol on information that solve Problem 1 and leads to the same result that in the centralized case.

\section{DeCentralized Protocols}

In order to obtain a distributed protocol $\Pi$ such that $A_{\Pi}=A$ it would be sufficient that the $i^{\text {th }}$-node could estimate $\tilde{l}$ and consequently to make the decision of ordering if $l_{i} \leq \tilde{l}$. A way to realize this is connected to the possibility of evaluating in a decentralized setting $\left|P_{l}\right|$ for each $l$ and hence $\tilde{l}$ from 1 .

\section{A. Linear Consensus Protocol}

We indicate with $L_{i}$ the $i^{\text {th }}$-row of the Laplacian Matrix of the graph.

\section{Theorem 2}

For fixed $l$, the distributed protocol $\Gamma$ defined by

$$
\left\{\begin{array}{cl}
h_{i}\left(x_{i}\right) & =x_{i} \\
f_{i}\left(y, x_{i}\right) & =-L_{i \bullet} x \\
\phi\left(x_{i}\right) & =n x_{i} .
\end{array}\right.
$$

where the $i^{t h}$-agent initializes its state component

$$
x_{i}(0)= \begin{cases}1 & \text { if } i \in P_{l} \quad \text { ("everyone orders") } \\ 0 & \text { if otherwise ("no one orders") }\end{cases}
$$

is such that all the local estimation of the cardinality of the set of active buyers converges asymptotically to the exact value, i.e. for the $i^{t h}$-agent it holds

$$
\lim _{t \rightarrow \infty} \phi_{i}\left(x_{i}\right)=\left|P_{l}\right|
$$

Proof: $\Gamma$ is an average consensus protocol. Thus, the $i^{t h}$

information vector is guaranteed to converge to the average computed on the initial values [7], namely,

$$
\bar{x}_{i}:=\lim _{t \rightarrow \infty} x_{i}=\operatorname{Ave}(x(0))
$$

We limit ourselves to note that

$$
\left|P_{l}\right|=\sum_{i=1}^{n} \hat{x}_{i}(0)=n \cdot \operatorname{Ave}(\hat{x}(0)) .
$$

Now, from definition (2) and (4-5) we prove (10). 
At each time instant the information vector can be interpreted as the local current estimation based on the available information up to the current instant time, of the percentage of nodes belonging to the set $P_{l}$. Initially, since information has not been exchanged yet, each agent estimates simply that all agents behaves in the same way as he does.

Converging properties of Protocol $\Gamma$ entail the consideration of a new protocol for the solution of Problem 1, defined as

$$
\left\{\begin{aligned}
h_{i}\left(x_{i}\right) & =x_{i} \\
f_{i}\left(y, x_{i}\right) & =\left\{-L_{i \bullet} x_{l \bullet}^{\prime}\right\}^{n} \quad l=1,2, \ldots, n \\
\phi\left(x_{i}\right) & =\max \left(l: n x_{l i}=l\right) .
\end{aligned}\right.
$$

where the $i^{t h}$-agent initializes the $l^{\text {th }}$-component of its initial state $x_{i}(0)=\{0,1\}^{n}$ according to

$$
x_{l i}(0)=\left\{\begin{array}{lll}
1 & \text { if } & i \in P_{l} \quad \text { ("everyone orders") } \\
0 & \text { if } & \text { otherwise ("no one orders") }
\end{array}\right.
$$

which is proved to verify $A_{\Pi}=A$.

This protocol allows to solve in parallel $n$ average consensus problems, one for each possible value of the threshold, $l$. Hence, it holds $m=n$ and this represent the main drawback of the protocol. Let us observe that other distributed protocols $\Pi$ with $A_{\Pi}=A$ and $m=n$ can be defined. However, we have introduced the previous one because its structure will turn to be useful for the sequel.

It is possible to reduce $m=n=1$ by running consensus protocols in sequence for successive threshold values. All this protocol are optimal with respect to Problem 1.

A possible consensus protocol is generated by the following algorithm:

Algorithm 1

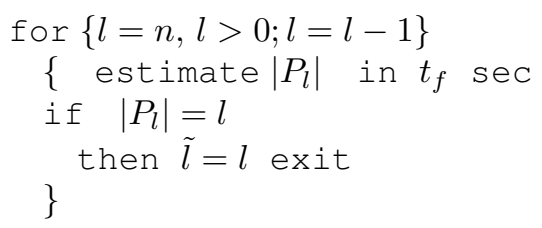

This protocol may converge in a fewer number of steps as it is evident from the following remark

\section{Remark -}

If at the $l^{t h}$-iteration it holds $\left|P_{l}\right|=r$ with $r<l$, all sets $\left|P_{s}\right|$ with $r<s<l$ have cardinality $\left|P_{s}\right| \leq r<s$ which means $\tilde{l}<s$ and therefore $P_{s}$ are not optimal.

\section{Example}

If $\left|P_{9}\right|=5$ then is certainly $\left|P_{8}\right| \leq 5,\left|P_{7}\right| \leq 5,\left|P_{6}\right| \leq 5$, and it must be checked the solution $\left|P_{5}\right|=5$.

Then an equivalent algorithm is the following, that requires fewer step.

\section{Algorithm 2}

$$
\begin{aligned}
& g=1 \\
& \text { for }(l=n ; l>0 ; l=l-g) \\
& \quad\left\{\text { estimate }\left|P_{l}\right|\right. \\
& \quad \text { if }\left|P_{l}\right|=l \text { then } \tilde{l}=l \text { exit } \\
& \quad g=l-\left|P_{l}\right| \\
& \quad\}
\end{aligned}
$$

In Example at the $1^{\text {st }}$-iteration one estimates $\left|P_{\mathcal{V}}\right|=5$; at the $2^{\text {nd }}$-iteration one must estimate $\left|P_{5}\right|$.

In the following subsection a possible implementation of the above described algorithm is introduced.

\section{B. Nonlinear Consensus Protocol}

In the proposed protocol we set $l=n$. The $i^{\text {th }}$-buyer communicates its initial estimation about the percentage of active buyers, $x_{i}$ to the neighbors. Then he updates it on-line on the basis of new estimates data received from neighbors. At any time, $t_{i}$, whenever the number of active buyers, $\phi_{i}\left(x_{i}\right)$, goes below its threshold, $l_{i}$, it leaves the group and communicate its decision to "give up" ordering by activating an exogenous impulse signal, $\delta_{i}\left(t-t_{i}\right)$. This exogenous impulse is activated every $t_{f}$, where $t_{f}$ is an estimate of the worst case possible settling time, corresponding to the worst possible topology of the network, i.e., a chain graph. The settling time is then related to the algebraic connectivity: $1 / \lambda_{2}(G)$, i.e., the second smallest eigenvalue of the Laplacian corresponding to a chain of $\mathcal{V}$ nodes. Theorem 3

Set $l=n$, the distributed protocol $\Pi$ defined by

$$
\left\{\begin{aligned}
h_{i}\left(x_{i}\right)= & x_{i} \\
f_{i}\left(y, x_{i}\right)= & -L_{i \bullet} x-\delta_{i}\left(t-t_{i}\right) \cdot 1\left[\left(n x_{i}\left(t_{i}\right)<l_{i}\right)\right. \\
& \operatorname{AND}\left(x_{i}(0) \neq 0\right) \\
& \left.\operatorname{AND}\left(t_{i}=k t_{f}, k \in \mathcal{N}\right)\right] \\
\phi\left(x_{i}\right)= & n x_{i} .
\end{aligned}\right.
$$

where $1[\gamma]$ a boolean function which returns the value 1 when condition $\gamma$ is true, 0 otherwise, and where each $i^{t h}$-agent initializes its state component

$$
x_{i}(0)=\left\{\begin{array}{lll}
1 & \text { if } & i \in P_{n} \quad \text { ("everyone orders") } \\
0 & \text { if } & \text { otherwise ("no one orders") }
\end{array}\right.
$$

is such that all local estimations of the cardinality of the set of active buyers converge asymptotically to the exact value, i.e., for each $i^{t h}$-agent it holds

$$
\lim _{t \rightarrow \infty} \phi_{i}\left(x_{i}\right)=|A|
$$

Proof: The presence of an exogenous negative impulse signal does not influence the dynamics of the system, but it acts as if the system evolves from a new initial state value anytime it occurs. Thus, when an impulse occurs at the $j^{t h}$ node, at instant $t_{i}$, it yields to a new initial condition where: $x_{j}\left(t_{i}^{+}\right)=x_{j}\left(t_{i}^{-}\right)-1$. (Clearly, the evolution starting at time $t_{i}$ has Laplace Transform $(s I+L) X(s)=x\left(t_{i}^{-}\right)-1$ and the new initial state value is $x\left(t_{i}^{+}\right)$). The system dynamics is the Laplacian $-L$ with $n-1$ real stable eigenvalues and one zero eigenvalue, and the zero-input response of the system converges to the Ave $(x(0))=\operatorname{Ave}(x)$ that is invariant if no 
impulse occurs, as shown in [7]. Because of the exogenous negative impulse signal, Ave $(x)$ is no longer an invariant quantity. It is evident that the final convergence value depends on how many impulse signals occur. If at time $t_{i}=t_{f}$, only one impulse occurs, we get $\operatorname{Ave}(x(t))=\operatorname{Ave}\left(x\left(t_{i}^{+}\right)\right)=$ $\operatorname{Ave}\left(x\left(t_{i}^{-}\right)\right)-1 / n=\operatorname{Ave}(x(0))-1 / n$.

In particular, the starting value $x_{j}(0)$ for each $j$ node is 0 or 1 , see (7). An impulse corresponds to a buyer which decides not to order anymore, then it occurs only for buyers whose initial value was 1 . The average value after successive impulses is monotonic decreasing and lower bounded by zero.

$$
\operatorname{Ave}(x)^{\prime}>\operatorname{Ave}(x)^{\prime \prime}>\ldots \geq 0
$$

Only when all the buyers were active (all ones in $x(0)$ ) at the beginning and they all decide not to order anymore, we reach the lower bound: Ave $(x(\infty))=0$.

Clearly, every time that a buyer drops off his order the average final value is decreased by one, then the final value is $\operatorname{Ave}(x)=\operatorname{Ave}(x(0))-\frac{M}{n}$ where $M$ is the total number of buyers having decided to drop off the order and so:

$$
\lim _{t \rightarrow \infty} \phi_{i}\left(x_{i}\right)=n \operatorname{Ave}(x(0))-M
$$

Consider now, a possible implementation of Algorithm 2, where at time instant 0 the $i^{t h}$-agent initializes its state component as in (7), and, to start estimating the cardinality of $P_{l}$ at the generic $\left(k^{t h}-1\right)$-iteration, let the agents reset their state $x_{i}\left(k t_{f}^{+}\right)=1$ if their threshold $l_{i} \leq l, 0$ otherwise. Hence, it holds $x_{i}\left(k t_{f}^{+}\right) \neq x_{i}\left((k-1) t_{f}^{+}\right)$for the agents $i$ with $l_{i} \geq l, x_{i}\left(k t_{f}^{+}\right)=x_{i}\left((k-1) t_{f}^{+}\right)$otherwise. Let us observe that at instant $k t_{f}^{-}$it holds

$$
\begin{aligned}
\operatorname{Ave}\left(x\left(k t_{f}^{-}\right)\right) & =\operatorname{Ave}\left(x\left((k-1) t_{f}^{+}\right)\right) \\
& =\frac{\sum_{i} x_{i}\left((k-1) t_{f}^{+}\right)}{n}=\frac{\sum_{i} x_{i}\left(k t_{f}^{-}\right)}{n}
\end{aligned}
$$

whereas, after the agents reset their state it holds

$$
\operatorname{Ave}\left(x\left(k t_{f}^{+}\right)\right)=\frac{\sum_{i} x_{i}\left(k t_{f}^{+}\right)}{n}=\frac{\sum_{i} x_{i}\left(k t_{f}^{-}\right)}{n}-\frac{M_{t_{f}}}{n}
$$

where $M_{t_{f}}$ is the number of agents $i$ such that $x_{i}\left(k t_{f}^{+}\right) \neq$ $x_{i}\left((k-1) t_{f}^{+}\right)$with threshold less than $l$. Note now that the same value of the left-hand term in (12) could be obtained if at time instant $k t_{f}^{+}$, the agents $i$ with threshold $l_{i} \leq l$ keep their state $x_{i}\left(k t_{f}^{+}\right)=x_{i}\left(k t_{f}^{-}\right)$whereas agents with threshold $l_{i} \geq l$ are required to activate the impulse so that $x_{i}\left(k t_{f}^{+}\right)=$ $x_{i}\left(k t_{f}^{-}\right)-1$. On the bases of what have been said, it is apparent that protocol $\Pi$ performs like Algorithm 2.

The above theorem has shown that $\left|A_{\Pi}\right|=|A|$. In addition, observe that in this case $m=n=1$. Note that this protocol is more suitable for application because at each instant $k t_{f}$ the only agents which are required to activate a control are those that are no longer interested in coordinating orders. Hence, even this protocol is optimal for Problem 1.

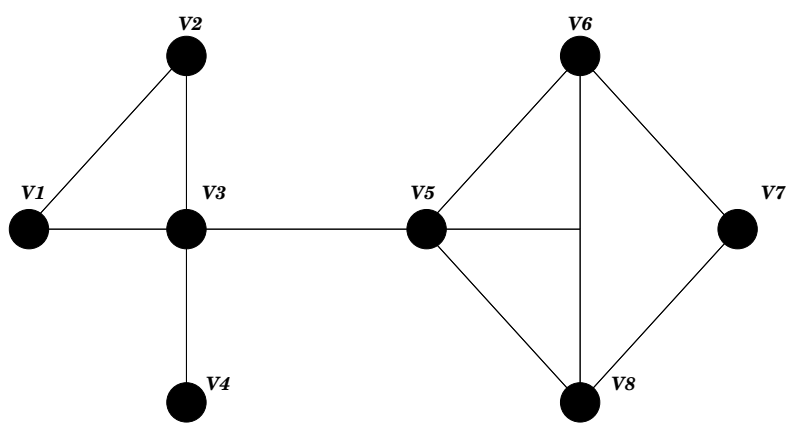

Fig. 1. Decentralized Information Structure

\section{Simulation SET-UP}

We considered a network of 8 agents and the associated information structure as in Fig. 1.

The components of the initial state vector $x(0)$ are randomly extracted from a uniform distribution in zero and one. The vector of thresholds is randomly extracted between one and eight. Thresholds associated to non active buyers are set equal to infinity. In particular the realization of both vector are shown in the table below

\begin{tabular}{|c|c|c|c|c|c|c|c|c|}
\hline agents & 1 & 2 & 3 & 4 & 5 & 6 & 7 & 8 \\
\hline$x_{i}(0)$ & 1 & 0 & 0 & 0 & 1 & 1 & 1 & 1 \\
\hline$l_{i}$ & 6 & $\infty$ & $\infty$ & $\infty$ & 3 & 2 & 5 & 2 \\
\hline
\end{tabular}

The information flow among agents is governed by the Laplacian that in this case takes on the form

$$
L=\left[\begin{array}{rrrrrrrr}
2 & -1 & -1 & 0 & 0 & 0 & 0 & 0 \\
-1 & 2 & -1 & 0 & 0 & 0 & 0 & 0 \\
-1 & -1 & 4 & -1 & -1 & 0 & 0 & 0 \\
0 & 0 & -1 & 1 & 0 & 0 & 0 & 0 \\
0 & 0 & -1 & 0 & 3 & -1 & 0 & -1 \\
0 & 0 & 0 & 0 & -1 & 3 & -1 & -1 \\
0 & 0 & 0 & 0 & 0 & -1 & 2 & -1 \\
0 & 0 & 0 & 0 & -1 & -1 & -1 & 3
\end{array}\right]
$$

Simulation of the protocol in Fig. 2 shows that at the beginning there is agent $1-5-6-7-8$ that intend to order. After $t_{f}=20 \mathrm{sec}$ all local estimations $\phi\left(x_{i}\right)$ of the number of active buyers are supposed to converge to 5 . Now, agent 1 give up because the estimation is lower than his corresponding threshold. Then at $t_{f}=40 \mathrm{sec}$ the new converging value is $\phi\left(x_{i}\right)=4$ and agent 7 gives up ordering. Finally the estimated number of active buyers is $\phi\left(x_{i}\right)=3$.

\section{CONCLUSION}

In this paper we propose a distributed consensus protocol for a network of buyers aiming at coordinating their ordering strategies.

In a centralized setting any buyer communicates to everyone 

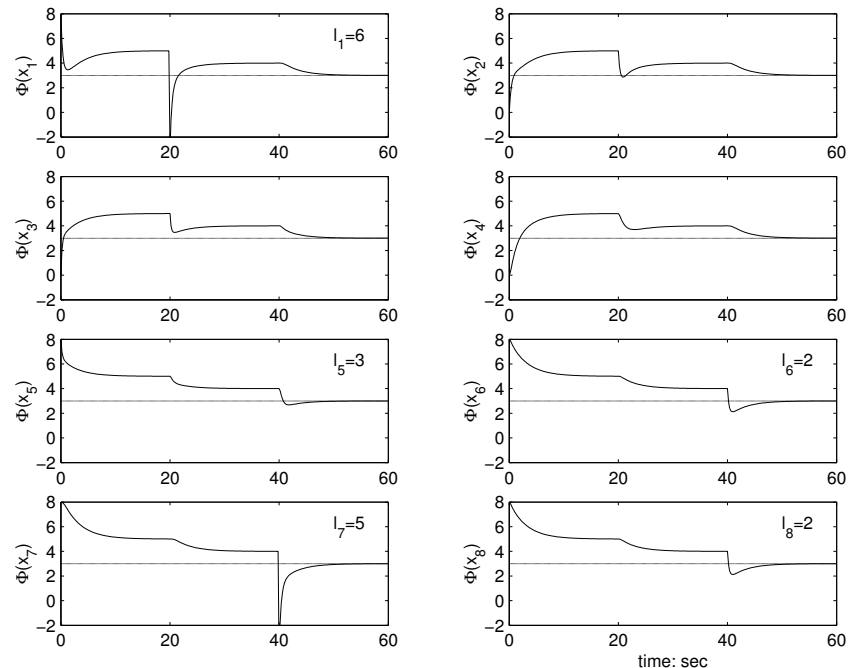

Fig. 2. Consensus for a group of 8 retailers

its desired threshold on the minimum number of active buyers to coordinate orders with. A centralized protocol processes all exchanged data and coordinates orders.

Alternatively, coordination can be achieved in a decentralized setting without the necessity that each buyer communicates the threshold. The method is based on a local estimation of the percentage of active buyers. The problem is formalized as an average-consensus problem. Thus, at the beginning of the day each buyer exchanges information with its neighbors regarding its initial local estimate (this initial information embeds its initial intention to order or not). Information propagates in a decentralized setting and converges to a common decisionvalue on the estimate within a pre-specified time interval. Once convergence is reached the current active buyers synchronize their new decision to give up ordering if the decision-value is lower than their threshold. We show that consensus on the final number of active buyers is reached globally asymptotically and coordination is the best achievable for the assigned thresholds.

\section{ACKNOWLEDGMENT}

This work is supported in part by the National Project PRIN: "Robust Techniques for the Control of Uncertain Systems" and by the National Research Council of Italy (CNR) under grant n. 00.00333.ST74.

\section{REFERENCES}

[1] F. Blanchini, S. Miani, W. Ukovich, "Control of Production-Distribution Systems with Unknown Inputs and Systems Failures" IEEE trans. on Automatic Control, vol 45, NO.6, June 2000

[2] A. Fax and R. M. Murray, "Information Flow and Cooperative Control of Vehicle Formations" The 15th IFAC World Congress, June 2002.

[3] A. J. Kleywegt, V. S. Nori, M. W. P. Savelsbergh, "The Stochastic Inventory Routing Problem with Direct Deliveries" Transportation Science, vol 36, No.1, 94-118, Feb 2002.

[4] S. H. Low, F. Paganini, J. C. Doyle, "Internet Congestion Control" IEEE Contr. Syst. Mag., vol 22, No.1, 28-43, Feb 2002.

[5] K. Passino, M. Polycarpou, D. Jacques, M. Pachter, Y. Liu, Y. Yang, M. Flint, and M. Baum, "Cooperative Control and Optimization, Chapter 12: Cooperative Control for Autonomous Air Vehicles, Kluwer Academic Publishers, 2002.
[6] R. O. Saber, W. B. Dunbar, R. M. Murray, "Cooperative Control of Multivehicle Systems using Cost Graphs and Optimization" submitted to the American Control Conference, June 2003.

[7] R. O. Saber and R. M. Murray, "Consensus Protocols for Networks of Dynamic Agents" Proc. of American Control Conference, Denver, Colorado, June 2003, www.cds.caltech.edu/ olfati/papers 